\begin{tabular}{|c|c|}
\hline Title & Photon A ntibunching Observed from an InA IA s Single Q uantum Dot \\
\hline Author(s) & $\begin{array}{l}\text { Kimura, Satoshi; Kumano, Hidekazu; Endo, Michiaki; Suemune, Ikuo; Y okoi, Tomonori; Sasakura, Hirotaka; A dachi, } \\
\text { Satoru; Muto, Shunichi; Song, Hai Zhi; Hirose, Shinichi; Usuki, Tatsuya }\end{array}$ \\
\hline Citation & $\begin{array}{l}\text { Japanese Journal of A pplied Physics. Pt. 2, Letters \& express letters, 44(25), L793 L } 796 \\
\text { https://doi.org/10.1143/JJA P.44.L793 }\end{array}$ \\
\hline Issue Date & 2005 \\
\hline Doc URL & http:/hdl .handle.net/2115/33088 \\
\hline Rights & Copyright $\odot 2005$ The Japan Society of A pplied Physics \\
\hline Type & article (author version) \\
\hline File Information & Kimura_JJAP_05.pdf \\
\hline
\end{tabular}

Instructions for use 


\title{
Photon Antibunching Observed from an InAlAs Single Quantum Dot
}

\author{
Satoshi KIMURA ${ }^{1}$, Hidekazu KUMANO ${ }^{1}$, Michiaki ENDO ${ }^{1}$, Ikuo SUEMUNE ${ }^{1,3}$, Tomonori \\ YOKOI $^{2}$, Hirotaka SASAKURA ${ }^{3}$, Satoru ADACHI ${ }^{2,3}$, Shunichi MUTO ${ }^{2,3}$, Hai Zhi SONG ${ }^{3,4}$, \\ Shinichi HIROSE ${ }^{5}$, Tatsuya USUKI ${ }^{3,4}$ \\ ${ }^{1}$ RIES, Hokkaido Univ. Sapporo 001-0021, Japan \\ ${ }^{2}$ Department of Applied Physics, Hokkaido Univ. Sapporo 060-8628, Japan \\ ${ }^{3}$ CREST JST, Kawaguchi 332-0012, Japan \\ ${ }^{4}$ Fujitsu Ltd., 10-1 Morinosato-Wakamiya, Atsugi 243-0197, Japan \\ ${ }^{5}$ Fujitsu Labs. Ltd., 10-1 Morinosato-Wakamiya, Atsugi 243-0197, Japan
}

\begin{abstract}
Single-photon emitters and detectors are the key devices to realize secure communications with single-photon-based quantum cryptography and single-photon-based quantum computing. InAlAs quantum dots (QDs) cover the wavelength range with high quantum efficiencies of Si-based single-photon detectors. Clear photon antibunching was observed from an InAlAs single QD under weak excitations. To realize single-photon emitters on demand, complete population of the QD energy states before the photon emission events is necessary, but the measured antibunching properties were dependent substantially on the photo-excitation powers. The physical origin of this problem is discussed and the criterion to distinguish the real deviation from the photon antibunching condition and the artifact of the measurements is clarified. The capability of single-photon emissions on demand will be demonstrated with photon antibunching under pulsed operations.
\end{abstract}




\section{Introduction}

Single-photon-based applications such as quantum cryptography and quantum computing are attracting much attention for realizing much more secure communications or ultra fast parallel computations. Recent progress of computer performances and future realization of quantum computers will endanger the security of present cryptography ${ }^{1)}$ and single-photon-based quantum cipher is expected to be an alternative cipher due to its unbreakable cryptography principle based on the indivisibility and noncloning properties of photons. $^{2)}$

Up to now, single-photon quantum ciphers have been achieved mostly with attenuated semiconductor laser sources. However because coherence of laser sources inherently results in the Poisson distributions of emitted photon numbers, laser lights have to be attenuated to the average photon numbers of $1 / 10$ or less to keep practical single-photon emission conditions. This results in serious problems such as low photon emission rates, random intervals of photon emission events, finite probabilities of emitting more than single photon, and higher bit-error rates. ${ }^{3)}$

To realize high-bit-rate secure communications single-photon sources, which deterministically provide single photons on demand, and photodetectors with high quantum efficiencies are inevitable. Single-photon emissions have been demonstrated with single atoms, single molecules, nitrogen-vacancy centers in diamonds, and single quantum dots (QDs). ${ }^{4)}$ Among them, semiconductor QDs have advantages in terms of their stability, high photon emission rates, and current-driving capabilities based on well-established semiconductor technologies. Silicon single-photon detectors have high quantum efficiencies in the wavelength range of $600-800 \mathrm{~nm}$ and the combination with QDs covering this wavelength range will be attractive for applications such as single-photon-based free-space quantum cryptography and quantum computing. ${ }^{5)}$ Some of the QD candidates will be $\mathrm{CdSe} / \mathrm{ZnS} \mathrm{QDs}^{6)}$ and InP QDs/InGaP ${ }^{7)}$ which cover the wavelength range around $580 \mathrm{~nm}$ and $700 \mathrm{~nm}$, respectively. The other potential candidate will be InAlAs QDs/AlGaAs, which cover the wavelength range around $750 \mathrm{~nm},{ }^{8)}$ and the photon antibunching needs to be proved.

In this paper, photon correlation measurements were performed on an InAlAs single QD and clear photon antibunching, i.e. the demonstration of single-photon emission, was observed with weak photo-excitation powers. However the properties were dependent on the excitation power and substantial degradation of the photon antibunching was observed with the increased excitations. It will be demonstrated that this phenomenon is due to the mixture of the artifact of the measurement setup and real degradation of the photon antibunching. The criterion to distinguish the two will be discussed and clarified. The capability of single-photon 
emissions on demand will be demonstrated with photon antibunching under pulsed operations.

\section{Experimental}

The QDs sample was grown on a (001) GaAs substrate by molecular-beam epitaxy. It had two-stacks of QDs layers made of $\operatorname{In}_{0.75} \mathrm{Al}_{0.25} \mathrm{As}$ and $\mathrm{In}_{0.7} \mathrm{Ga}_{0.3} \mathrm{As}$ separated by an 11-nm-thick $\mathrm{Al}_{0.3} \mathrm{Ga}_{0.7}$ As layer. ${ }^{8)}$ These QDs were prepared in Stranski-Krastanow growth mode on $\mathrm{Al}_{0.3} \mathrm{Ga}_{0.7} \mathrm{As}$ layers and were sandwiched with $\mathrm{Al}_{0.3} \mathrm{Ga}_{0.7} \mathrm{As}$ layers. The topmost surface was terminated with a GaAs cap layer. After the growth, the sample was etched into mesa structures with diameters of $\sim 150 \mathrm{~nm}$ for single QDs measurements. The details of this sample preparation are described in Ref. 8. The emission wavelengths of the $\operatorname{In}_{0.75} \mathrm{Al}_{0.25} \mathrm{As}$ and $\mathrm{In}_{0.7} \mathrm{Ga}_{0.3}$ As QDs are around the wavelengths of $750 \mathrm{~nm}$ and $950 \mathrm{~nm}$, respectively and the coupling effects of the two-kinds of QDs in the neighboring layers were almost negligible with the 11-nm-thick $\mathrm{Al}_{0.3} \mathrm{Ga}_{0.7}$ As spacer layer, ${ }^{9)}$ and this work was focused on the former $\mathrm{In}_{0.75} \mathrm{Al}_{0.25} \mathrm{As}$ QDs with the shorter emission wavelength.

In our measurements, the sample was held in a closed-loop He cryostat and was kept at 23K. A He-Ne laser at the wavelength of $632.8 \mathrm{~nm}$ was used for continuous-wave (CW) excitations and a mode-locked pulsed Ti: sapphire laser at the wavelength of $730 \mathrm{~nm}$ with the pulse width of $80 \mathrm{fs}$ and the pulse repetition frequency of $82 \mathrm{MHz}$ was used for pulsed excitations. The laser power was attenuated by variable neutral-density filter to control the excitation condition. An objective lens with the numerical aperture (NA) of 0.5 focused the laser beam on one of the mesa structures and collected luminescence emitted from several QDs in the mesa. The excitation power was measured in front of the objective lens in this work, and therefore the actual excitation power will be lower than the values given in the following graphs. Collected luminescence was dispersed by a $0.5-\mathrm{m}$ spectrometer and was detected with Si charge-coupled-device detectors in spectral measurements.

The verification of single-photon emission at a time, i.e. photon antibunching was examined with photon correlation measurements employing Hanbury-Brown and Twiss (HBT) setup. ${ }^{10)}$ Only an exciton peak of a single QD was selected by a $0.2-\mathrm{m}$ monochromator and emitted photons were separated into two paths by a nonpolarized beam splitter. Photons incident in each path were detected by two single-photon-counting modules (SPCMs). After the photon detections electrical pulses were sent from the two SPCMs in each path to a time-amplitude converter (TAC), and the TAC generated a voltage proportional to the time delay between the two pulses from the two SPCMs. A multi-channel-analyzer (MCA) assigned a channel corresponding to the time delay depending on the voltage generated by the 
TAC and gave an output count in the channel, which accumulated into a histogram. In the low count rate limit of the SPCMs, where the average time between detected photons is much longer than the measured delay $\tau$, this measured coincidence count histogram is proportional to the second-order intensity correlation function, $\mathrm{g}^{(2)}(\tau)$, and therefore normalized histograms are equivalent to $\mathrm{g}^{(2)}(\tau){ }^{11)}$ If an incident photon is purely single and the probability to find another photon nearby is zero, i.e. ,antibunching, $g^{(2)}(0)$ will be equal to zero. ${ }^{12)}$

The photon correlation measurements are possible with both $\mathrm{CW}$ and pulsed excitations. The operations of single-photon emissions on demand will be possible if single photons are emitted after every pulsed excitations. On the other hand, CW excitations have the advantage to estimate simultaneously the exciton physical quantities such as recombination lifetimes under the same operating conditions in the photon correlation measurements. ${ }^{11)}$ In this paper, the photon antibunching properties will be discussed mainly with the $\mathrm{CW}$ measurements together with the pulsed operations to demonstrate the capability of single photon emissions on demand.

\section{3. $C W$ photon correlation measurements}

A photoluminescence (PL) spectrum measured from a single InAlAs QD with the He-Ne laser excitation power of $20 \mu \mathrm{W}$ is shown in Fig. 1. Sharp peak was observed at the photon energy of $1.6009 \mathrm{eV}$ as shown by the closed circles. Its full width at half maximum (FWHM) was about $200 \mu \mathrm{eV}$ and the spectrum was well fitted with the Lorentzian line-shape function given by the solid line. This indicates that this emission peak originates from a single radiative center. The integraged PL intensity was almost linearly dependent on the excitation power, $\mathrm{P}_{\mathrm{ex}}$, i.e. $\propto P_{e x}^{1.11}$ (not shown), and the origin of this PL peak was attributed to the exciton emission from a single InAlAs QD. The measured spectra were dominated by the exciton peak below the excitation power of $75 \mu \mathrm{W}$, and the biexciton peak at $1.5963 \mathrm{eV}$ was observed above that excitation power. The observed FWHM of $200 \mu \mathrm{eV}$ is broader than other reported values, but the linewidths varied from dot to dot within $30-200 \mu \mathrm{eV}$ on this sample. This is attributed to environmental effects on the broadening processes of radiative transitions in single QDs. ${ }^{13)}$

Photon emission processes from the QD were examined with the CW photon correlation measurements and the measured results with the excitation powers of $10 \mu \mathrm{W}$ and $75 \mu \mathrm{W}$ are shown by the insets in Fig. 2. The measured TAC time window was 50 ns and the time bin size was $\sim 100$ ps. The SPCMs total count rate was $1.5 \times 10^{4}$ count per second for the excitation power of $10 \mu \mathrm{W}$ and was higher for the higher excitations. The clear dip of $g^{(2)}(\tau)$ was 
observed at $\tau=0$ under the lower excitation power of $10 \mu \mathrm{W}$, which is closer to photon antibunching. Following the conventional fitting method of the measurement with the equation, 6,14$)$

$$
\mathrm{g}^{(2)}(\tau)=1-\beta \exp \left(-\tau / \tau_{\text {rise }}\right),
$$

$g^{(2)}(0)=1-\beta$ was estimated to be the low value of 0.05 . However as is shown in Fig. $2, g^{(2)}(0)$ increased substantially with the increase of the excitation power and $g^{(2)}(0)$ was estimated to be 0.42 with the excitation power of $75 \mu \mathrm{W}$ with the fit shown by the inset in Fig. 2 . This may suggest the break down of the photon antibunching state under the higher excitation of the QD. This will be a serious problem to realize single photon emitters on demand, since QD should be populated with high probability just before each photon emission event for its reproducible operations. Therefore full understanding of this excitation-power dependence is essential to realize realistic single-photon emitters.

The second-order intensity correlation function, $g^{(2)}(\tau)$, is proportional to the probability density of detecting another photon with the time delay $\tau$ after a photon was detected. This probability is proportional to $\mathrm{p}_{1}(\tau)$, the probability of finding the QD with one electron-hole pair, with the condition that the QD had zero electron-hole pairs at $\tau=0 .{ }^{11)}$ In this case, $p_{1}(\tau)$ is given by solving the following rate equations:

$$
\begin{aligned}
& \dot{p}_{0}(\tau)=-G p_{0}(\tau)+\frac{p_{1}(\tau)}{\tau_{1}}, \\
& \dot{⿱_{1}}(\tau)=G p_{0}(\tau)-\frac{p_{1}(\tau)}{\tau_{1}},
\end{aligned}
$$

where $p_{0}(\tau)$ is the probability finding the QD without any electron-hole pairs, $\tau_{1}$ is the recombination lifetime, and $\mathrm{G}$ is the excitation rate. The biexciton or two electron-hole pairs state was not included here for the mainly treated weak excitation regime. Using the condition of $\mathrm{p}_{0}+\mathrm{p}_{1}=1$ and with the initial conditions of $\mathrm{p}_{0}(0)=1$ and $\mathrm{p}_{1}(0)=0, \mathrm{p}_{1}(\tau)$ is derived as

$$
p_{1}(\tau)=\frac{G \tau_{1}}{G \tau_{1}+1}\left[1-\exp \left\{-\left(G+\frac{1}{\tau_{1}}\right) \tau\right\}\right] \propto g^{(2)}(\tau) .
$$

An additional analysis including two electron-hole pairs state was also performed and the derived expression for $\mathrm{p}_{1}(\tau)$ was shown to approach Eq. (3) under the weak excitation regime treated here (not shown). Equation (3) indicates that the rise rate $\left(1 / \tau_{\text {rise }}\right)$ of $g^{(2)}(\tau)$ has the excitation power dependence in the form of $1 / \tau_{\text {rise }}=G+1 / \tau_{1}$. However in this expression, $\mathrm{g}^{(2)}(0)$ remains zero regardless of the excitation power and Eq. (3) cannot explain the measured excitation power dependence of $g^{(2)}(0)$.

In the HBT measurement setup, the time resolutions of SPCMs, TAC, and MCA may affect the correlation measurements, especially the correlation dip, $g^{(2)}(0) .^{7,15)}$ Therefore this 
broadening of the measurement system was estimated by measuring the output response using the 80-fs-pulsed Ti:Sapphire laser as a delta-function-like input to the system. The system response was well fitted by the Gaussian function, $h(\tau)=(1 / \sqrt{2 \pi} \Delta t) \exp \left[-(\tau / \Delta t)^{2} / 2\right]$ (not shown), and the time broadening was estimated to be $\Delta \mathrm{t}=0.55 \mathrm{~ns}$. Taking into account this time response of the system, the measured correlation function is given by the convolution of a real $\mathrm{g}^{(2)}(\tau)$ and the system response given by the Gaussian function,

$$
g_{\text {meas }}^{(2)}(\tau)=\int_{-\infty}^{\infty} h\left(\tau-\tau^{\prime}\right) g^{(2)}\left(\tau^{\prime}\right) d \tau^{\prime}
$$

$\tau_{\text {rise }}=1 /\left(\mathrm{G}+1 / \tau_{1}\right)$ in Eq. (3) will be uniquely determined by fitting Eq. (4) to the measured correlation functions.

One example of the fitting is shown in Fig. 3 for the excitation power of $10 \mu \mathrm{W}$. The optimized fitting of the measurement with Eq. (4) is shown by the solid line, and $\tau_{\text {rise }}$ was estimated to be $1.88 \mathrm{~ns}$. The dashed line is the plot of $\mathrm{g}^{(2)}(\tau)$ of which $\tau$ dependence is given by Eq. (3). While $g^{(2)}(0)$ is equal to zero, $g_{\text {meas }}^{(2)}(0)$ is increased to 0.15 due to the broadening of the system response. It will be clear from the comparison of the solid and dashed lines in Fig. 3 that the characteristics near the zero delay time is much influenced by the system response as discussed above but the overall characteristics are not sensitively dependent on the system response and is uniquely determined by the rise time $\tau_{\text {rise. }}$. It is noted that the fitting with the conventional form given by Eq. (1) results in erroneous fitting of the $\tau_{\text {rise }}$ value.

The rise rates, $1 / \tau_{\text {rise }}$, estimated with the different excitation powers are plotted in Fig. 4(a). The characteristics showed the linear dependence on the excitation power as expected from the form of $1 / \tau_{\text {rise }}=G+1 / \tau_{1}$ and the recombination lifetime $\tau_{1}$ was estimated to be $2.3 \mathrm{~ns}$ from the vertical axis at the zero excitation power. The comparison with the direct lifetime measurements will be discussed later.

The $g^{(2)}(0)$ values calculated from Eq. (4) with the fitted $\tau_{\text {rise }}$ values under each excitation power were well fitted by the dot-dash line in Fig. 4(b) and showed the lowest limit of the $\mathrm{g}^{(2)}(0)$ value measurable with the present HBT setup, which is limited by the time broadening of the measurement system. The $g^{(2)}(0)$ values given by the fitting with Eq. (1), which were given in Fig. 2, are replotted by the open circles in Fig. 4(b). As is clear in Fig. 4(b), this fitting underestimates the $\mathrm{g}^{(2)}(0)$ values below the lowest limit for the weak excitation range. This is because the function given by Eq. (1) does not include the time broadening and is too sharp to fit the deep dip around the zero time delay. To examine the range where the open circles are above the lowest limit shown by the dashed line, the fittings of the measurements were performed by substituting Eq. (1) into the right-hand-side equation in Eq. (4) and the 
fitted $g_{\text {meas }}^{(2)}(0)$ values were shown by the closed squares. The comparison of the measured values with the lowest limit given by the dot-dash line demonstrates that the measured $\mathrm{g}^{(2)}(0)$ values were almost completely antibunched below the excitation power of $40 \mu \mathrm{W}$. The real $\mathrm{g}^{(2)}(0)$ values for the excitation powers of $75 \mu \mathrm{W}$ and $100 \mu \mathrm{W}$ were estimated to be 0.10 and 0.35 , respectively.

\section{Pulsed photon correlation measurements}

The capability of single-photon emissions on demand was examined with pulsed photon correlation measurements. The Ti:Sapphire laser was used for the excitation of the mesa structure. The measured TAC time window was $200 \mathrm{~ns}$ and the time bin size was $\sim 400 \mathrm{ps}$. The SPCMs total count rate was $8 \times 10^{3}$ count per second for the average excitation power of $3 \mu \mathrm{W}$. As is shown in Fig. 5, the clear dip of the coincidence count, $g^{(2)}(\tau)$, was observed at $\tau=0$, demonstrating the photon antibunching. The residual counts were limited by the background noise in the measurement setup.

\section{Discussions}

In Fig. 4(b) in the strong excitation regime above the excitation power of $\sim 60 \mu \mathrm{W}$, the increase of the $g^{(2)}(0)$ value was clearly observed. This range corresponded to the appearance of the biexciton peak and therefore this phenomenon will be related to multiexciton excitations. The background noise was also increased in this range and will be due to multi-exciton relaxations originated from the $\mathrm{Al}_{0.3} \mathrm{Ga}_{0.7} \mathrm{As}$ barrier-layer excitation by the He-Ne laser. This increase of the background noise will cause the increase of $g^{(2)}(0)$. This background noise issue may be reduced by resonant or quasiresonat excitations of the QDs. ${ }^{16)}$

The recombination lifetime estimated from Fig. 4(a) was $2.3 \mathrm{~ns}$ in the $\mathrm{CW}$ correlation measurements, while the lifetime measured directly with time-resolved-photoluminescence (TRPL) measurements with Ti:Sapphire laser was $1.12 \mathrm{~ns}$ (not shown). This discrepancy may be caused by the difference of the excitation photon energy. In the TRPL measurements, the $\mathrm{In}_{0.75} \mathrm{Al}_{0.25} \mathrm{As}$ wetting layer was excited at the photon energy of $1.698 \mathrm{eV}(\lambda=730 \mathrm{~nm})$, while in the $\mathrm{CW}$-excitation correlation measurements, the $\mathrm{Al}_{0.3} \mathrm{Ga}_{0.7} \mathrm{As}$ barrier layer was excited at the photon energy of $1.959 \mathrm{eV}(\lambda=632.8 \mathrm{~nm})$. This barrier-layer excitation in the $\mathrm{CW}$ measurement will result in longer effective recombination lifetimes due to the diffusion in the wider range of the $\mathrm{Al}_{0.3} \mathrm{Ga}_{0.7}$ As layers. ${ }^{16)}$

From the above discussions, the substantial excitation power dependence of the $\mathrm{g}^{(2)}(0)$ 
value is attributed to the excitation-power-dependent rise rate given by $1 / \tau_{\text {rise }}=\mathrm{G}+1 / \tau_{1}$ in Eq. (3) convolved with the measurement system response given by Eq. (4). This arises more crucially when the recombination lifetime $\tau_{1}$ is close or shorter than the time resolution of the system response such as in the present case. In case of $\mathrm{CdSe} / \mathrm{ZnS}$ nanocrystals, clear antibunching was observed in much wider operation conditions. ${ }^{11)}$ This is because the lifetime in this system is $\sim 20 \mathrm{~ns}$ and is much longer that the time broadening of the system and therefore the problem discussed in this paper is not much involved in the measurements. However for higher-bit-rate photon emissions, shorter lifetime is preferable and the problems discussed in this paper have to be considered inherently.

\section{Conclusions}

Clear photon antibunching was observed from the InAlAs single QD. Substantial excitation-power dependence of the $\mathrm{g}^{(2)}(0)$ value was observed, but the criterion to distinguish the real deviation from the photon antibunching condition and the artifact of the measurements was clarified, and the complete antibunching was demonstrated up to certain excitation power range. The capability of single-photon emissions on demand was demonstrated with photon antibunching under pulsed operations. The present InAlAs QDs cover the wavelength range with high quantum efficiencies of Si-based single-photon detectors and will be attractive for applications such as single-photon-based free-space quantum cryptography and quantum computing.

\section{Acknowledgment}

This work was supported in part by the Grant-in-Aid for Scientific Research (S) (2), No. 16106005 from the Ministry of Education, Science, Sports, and Culture. 


\section{References.}

1) Math world article http://mathworld.wolfram.com/news/2003-12-05/rsa/

2) N. Gisin, G. Ribordy, W. Tittel, and H. Zbinden, Rev. Mod. Phys. 74 (2002) 145.

3) D. S. Bethune, M. Navarro, and W. P. Risk, Appl. Optics 41 (2002) 1640.

4) for example, V. Zwiller, T. Aichele, and O. Benson, New J. Phys. 6 (2004) 96.

5) B. C. Jacobs and J. D. Franson, Opt. Lett. 21 (1996) 1854.

6) P. Michler, A. Imamoglu, M. D. Mason, P. J. Carson, G. F. Strouse, and S. K. Buratto, Nature 406 (2000) 968.

7) V. Zwiller, T. Aichele, W. Seifert, J. Persson, and O. Benson, Appl. Phys. Lett. 82 (2003) 1509.

8) T. Yokoi, S. Adachi, H. Sasakura, S. Muto, H. Z. Song, T. Usuki, and S. Hirose, Phys. Rev. B 71 (2005) 041307.

9) H. Sasakura, S. Adachi, S. Muto, H. Z. Song, T. Miyazawa and T. Usuki, Jpn. J. Appl. Phys. 43 (2004) 2110.

10) R. Hanbury-Brown and R. Q. Twiss, Nature 177 (1956) 27.

11) B. Lounis, H. A. Bechtel, D. Gerion, P. Alivisatos, W. E. Moerner, Chemical Physics Letters 329 (2000) 399.

12) R. Loudon, The Quantum Theory of Light (Oxford University Press. Oxford, New York, 2000), 3rd ed, chapter 9.

13) C. Kammerer, G. Cassabois, C. Voisin, M. Perrin, C. Delalande, Ph. Roussignol, and J. M. Gérard, Appl. Phys. Lett. 81 (2002) 2737.

14) V. Zwiller, H. Blom, P. Jonsson, N. Panev, S. Jeppesen, T. Tsegaye, E. Goobar, M.-E. Pistol. L. Samuelson, and G. Björk, Appl. Phys. Lett. 78 (2001) 2476,

15) A. Malko, M. H. Baier, E. Pelucchi, D. Y. Oberli, K. Leifer, D. Chek-al-kar, and E. Kapon, Appl. Phys. Lett. 85 (2004) 5715.

16) C. Santori, D. Fattal, J. Vukovi, G. S. Solomon, E. Waks, and Y. Yamamoto, Phys. Rev. B 69 (2004) 205324. 


\section{Figure captions}

Fig. 1. PL spectrum measured from a single InAlAs QD with the He-Ne laser excitation power of $20 \mu \mathrm{W}$.

Fig. 2. Measured excitation power dependence of $\mathrm{g}^{(2)}(0)$ values. Insets are $\mathrm{CW}$ photon correlation measurements at the excitation powers of $10 \mu \mathrm{W}$ and $75 \mu \mathrm{W}$.

Fig. 3. Fitting of photon correlation measurements for the excitation power of $10 \mu \mathrm{W}$. Solid line includes the system response, while dashed line is fitting without the system response.

Fig. 4. (a) Rise rates estimated with different excitation powers are plotted closed circles. The solid line is the fit with the form of $1 / \tau_{\text {rise }}=G+1 / \tau_{1}$. (b) $g^{(2)}(0)$ values derived with fitting without system time broadening (open circles), with system time broadening (closed squares). Dot-dash line shows the lowest limit of the $g^{(2)}(0)$ value measurable with the present HBT setup.

Fig. 5. Pulsed photon correlation measurements on the exciton emission from the InAlAs single QD. The Ti:Sapphire laser with the wavelength of $730 \mathrm{~nm}$ was used for the excitation of the mesa structure. 
Fig. 1 S. Kimura etal.

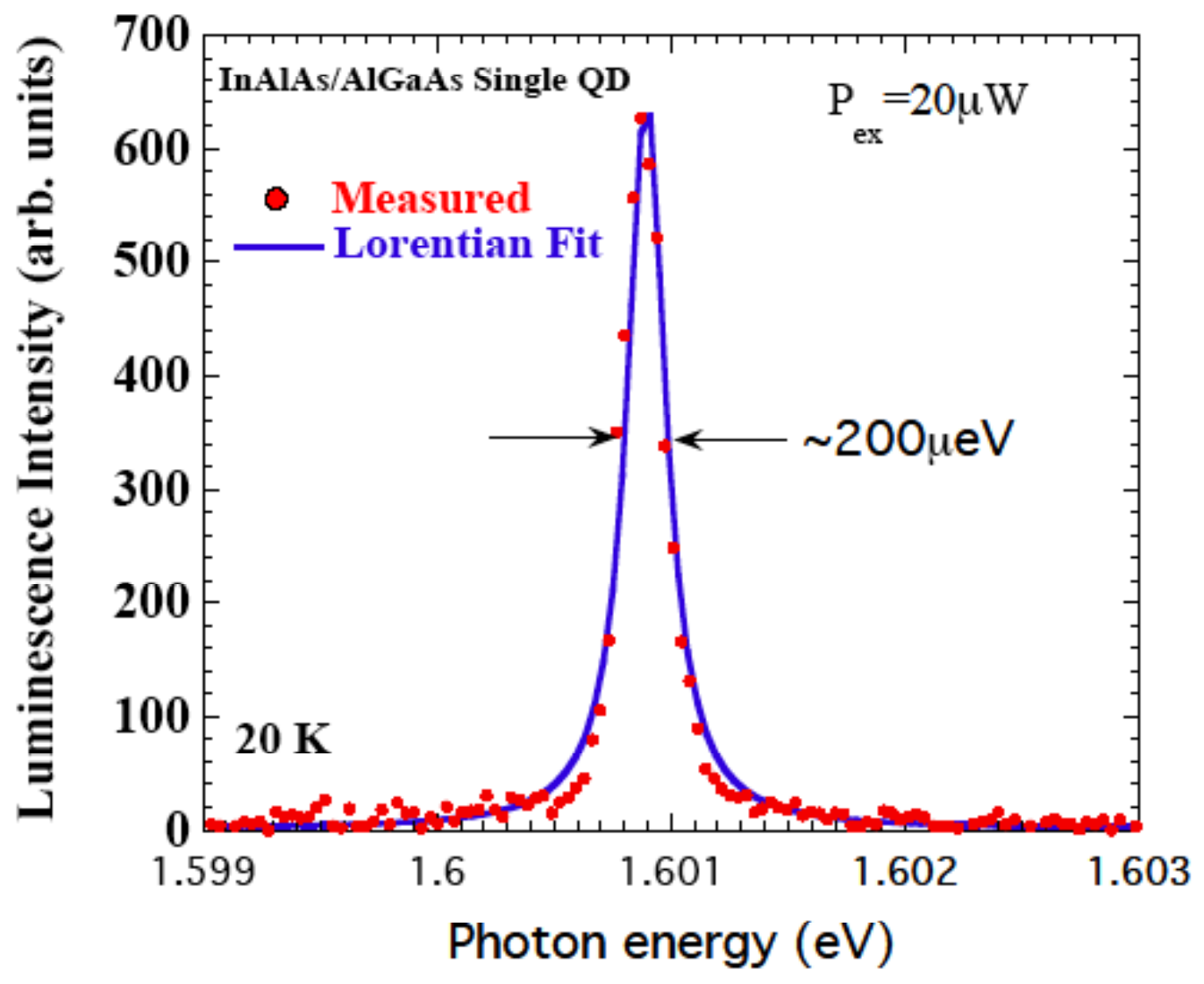


Fig. 2 S. Kimura etal.

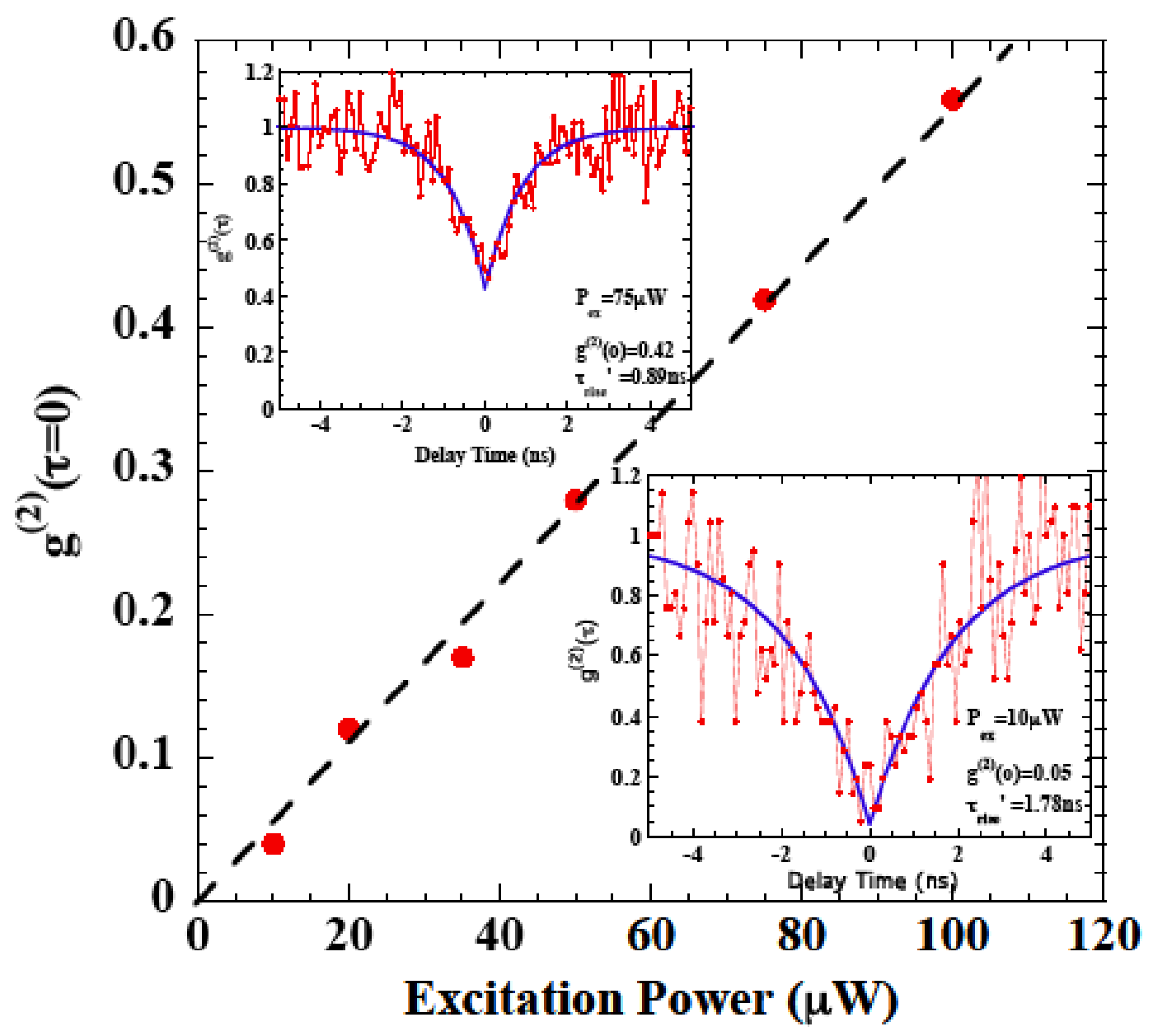


Fig. 3 S. Kimura etal.

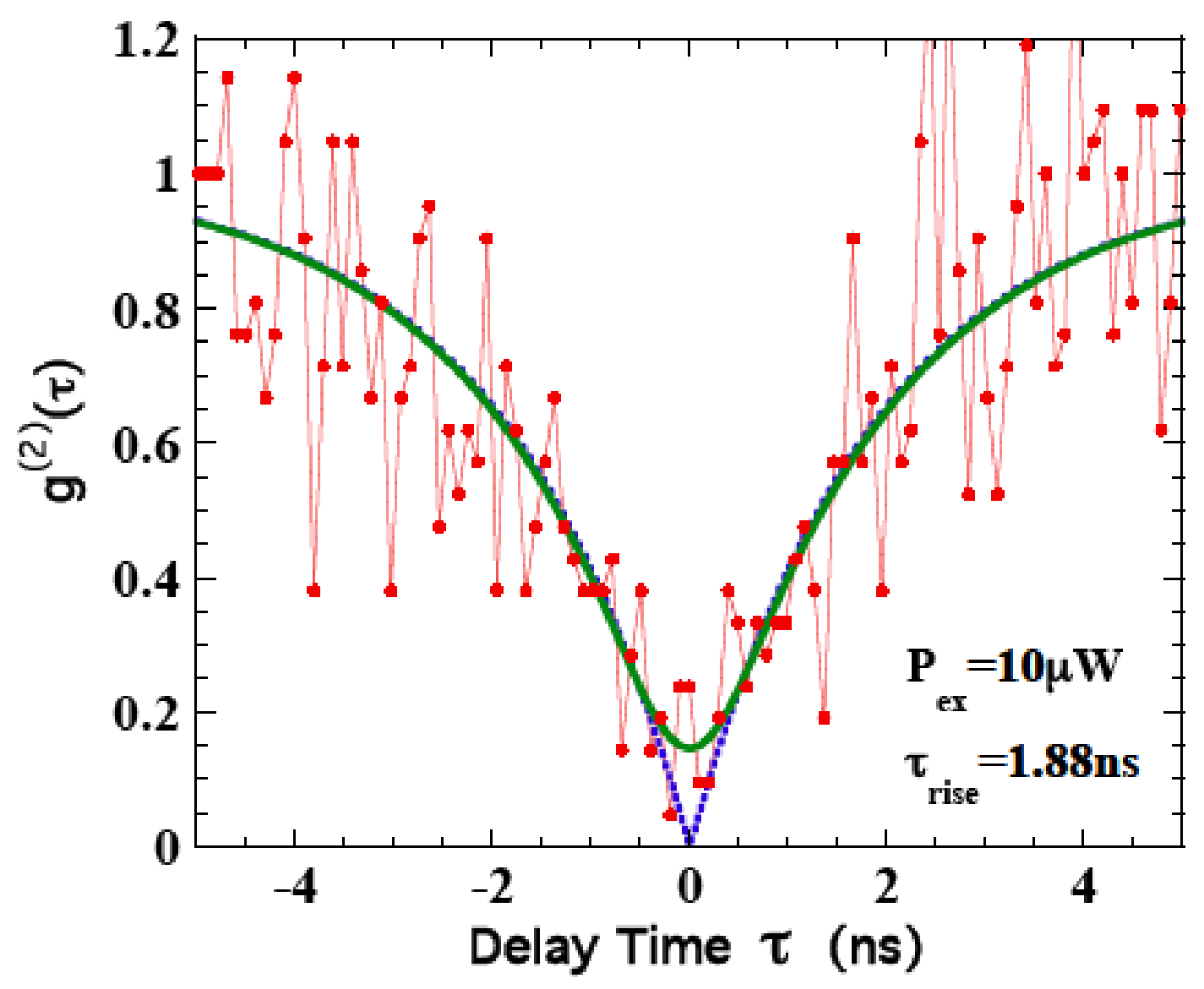


Fig. 4(a) S. Kimura etal.

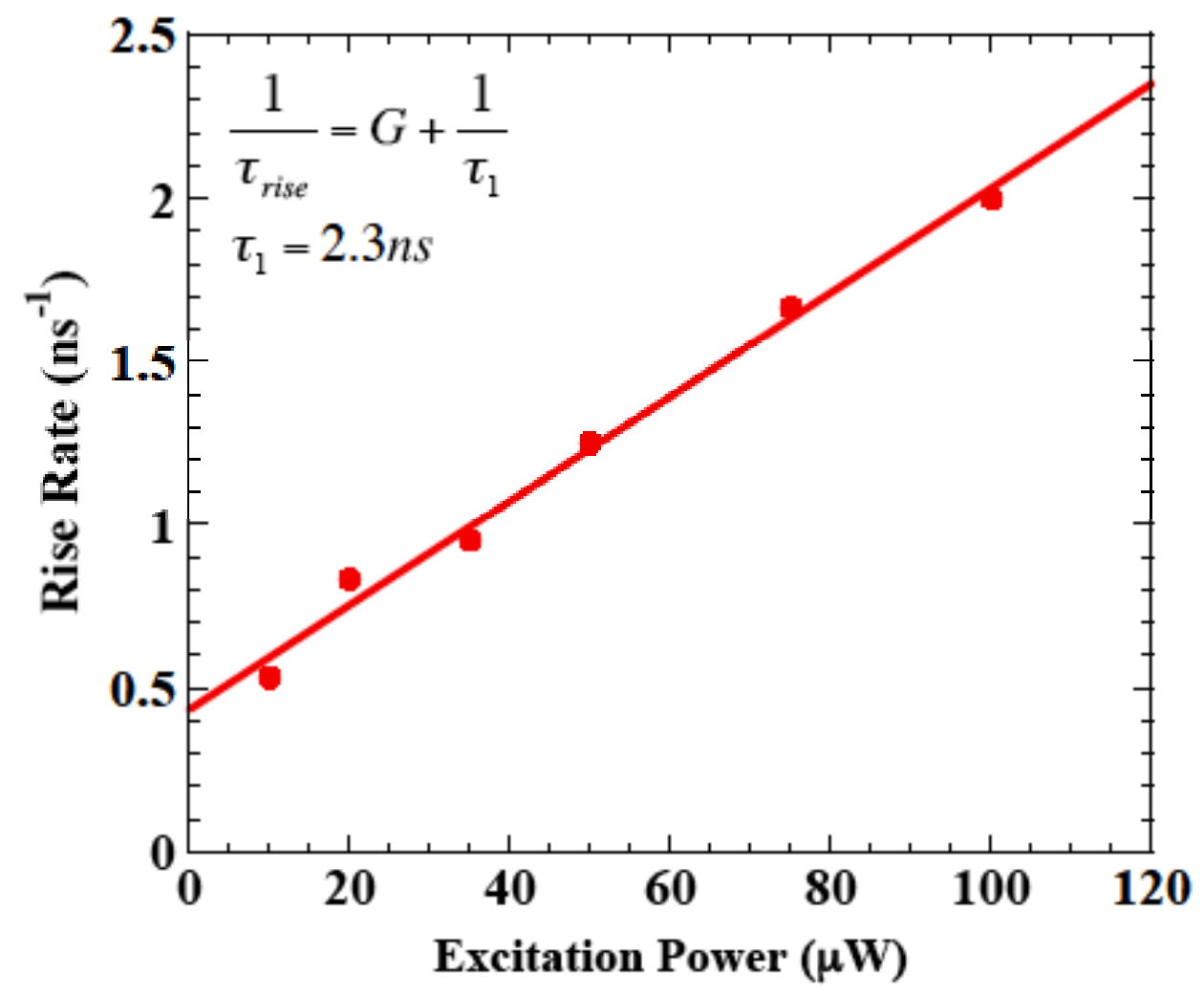


Fig. 4(b) S. Kimura etal.

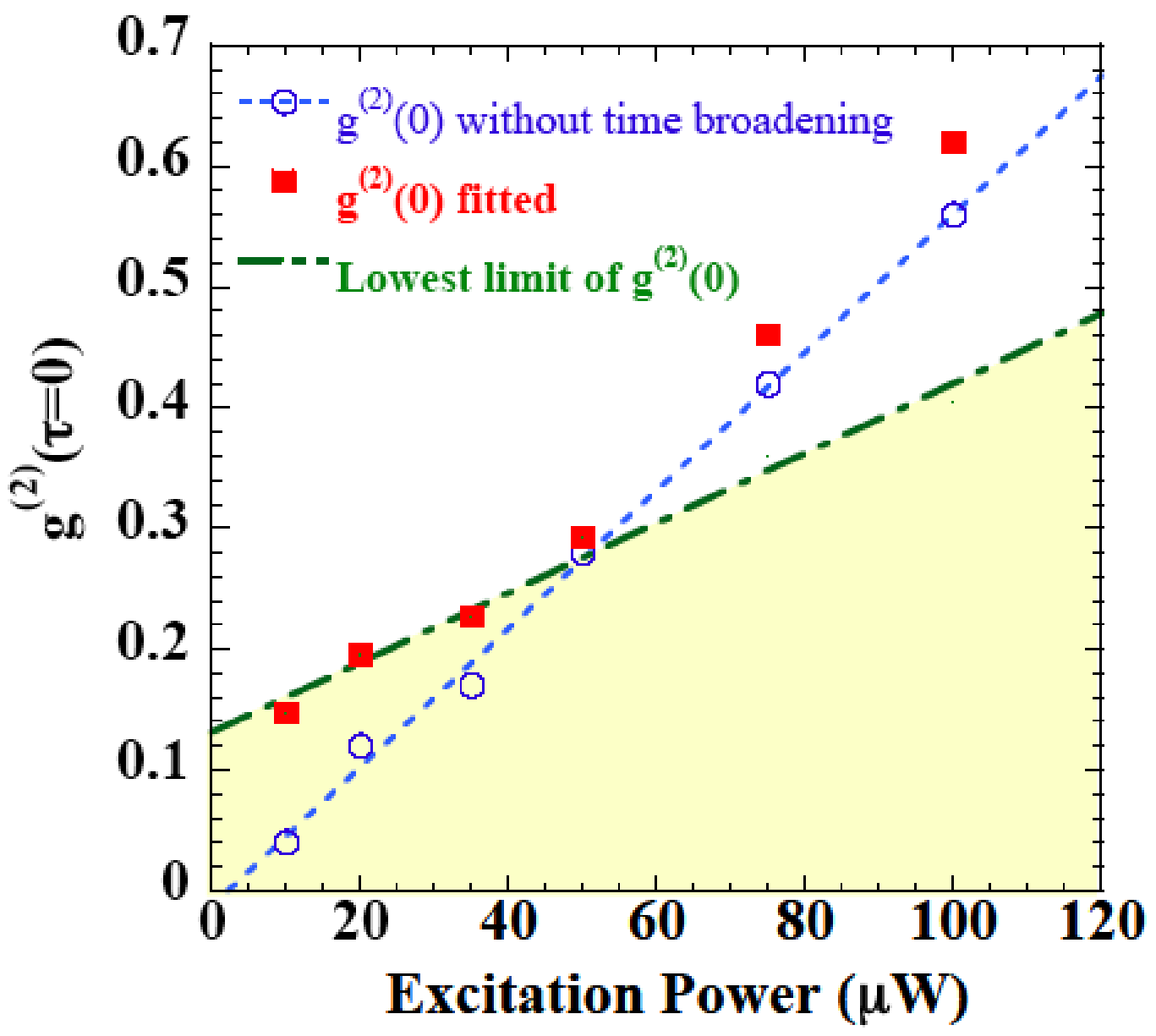


Fig. 5 S. Kimura etal.

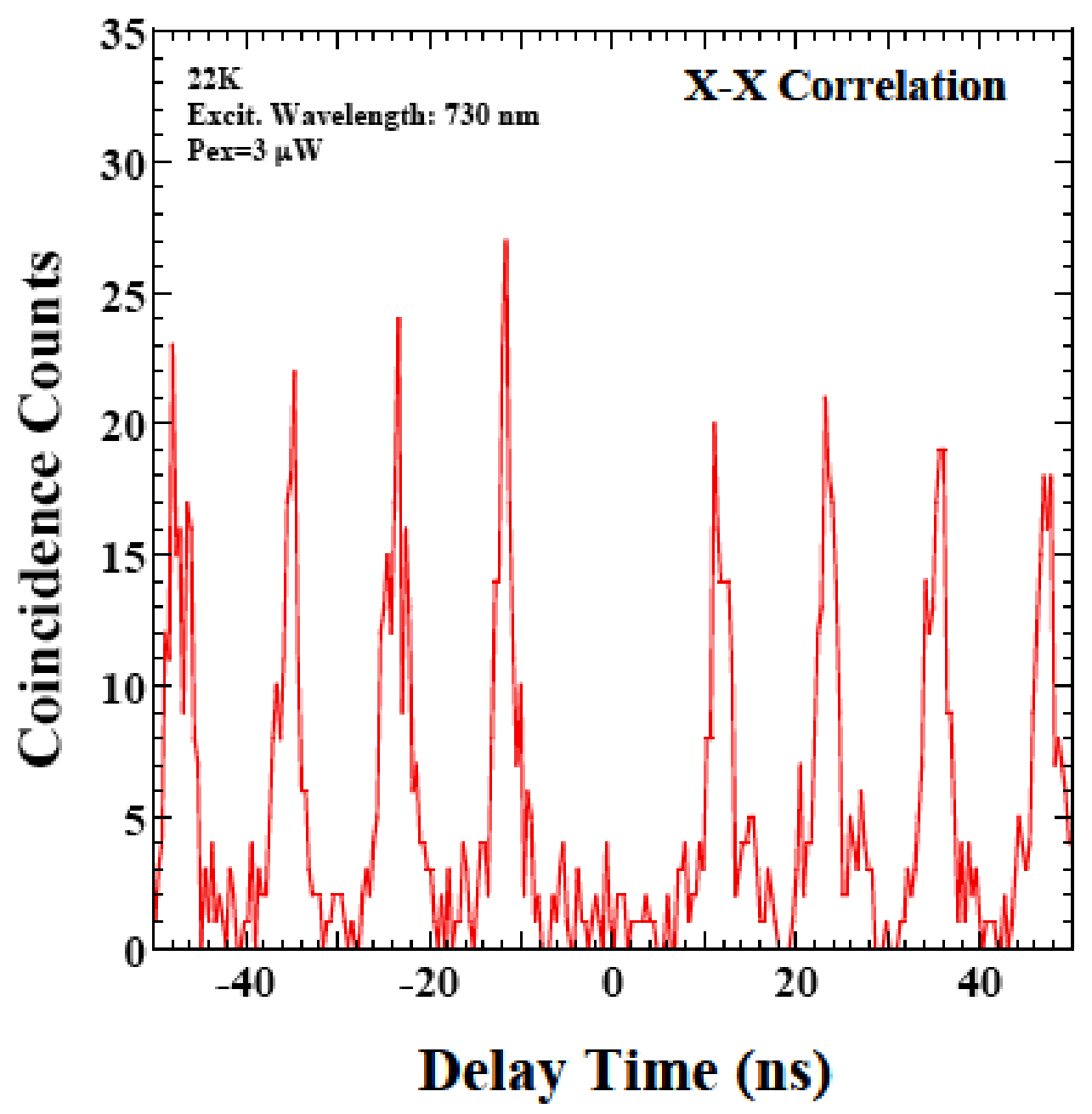

